\title{
Singular perturbations for phase transitions
}

\section{Margarida Baía}

Center for Nonlinear Analysis, Geometry and Dynamical Systems Departamento de Matemática, Instituto Superior Técnico 1049-001 Lisboa, Portugal

E-mail address: mbaia@math.ist.utl.pt

\section{Ana Cristina Barroso}

Universidade de Lisboa, Faculdade de Ciências and CMAF

Av. Prof. Gama Pinto 2, 1649-003 Lisboa, Portugal

E-mail address: abarroso@ptmat.fc.ul.pt

\section{José Matias}

Center for Nonlinear Analysis, Geometry and Dynamical Systems

Departamento de Matemática, Instituto Superior Técnico 1049-001 Lisboa, Portugal

E-mail address: jmatias@math.ist.utl.pt

Dedicated to Luís Magalhães and Carlos Rocha on the occasion of their 60th birthday

\begin{abstract}
We present a short overview of some singular perturbation problems that arise in the context of phase transitions.
\end{abstract}

\section{Contents}

1. Introduction

2. Notation and preliminaries

3. First order problems

4. Second order problems

2010 Mathematics Subject Classification. 49J40,49J45,49K20,74G65.

Key words: Two-Phase Transition Problems, Singular Perturbations, Functions of Bounded Variation, $\Gamma$-Convergence. 


\section{Introduction}

Our aim in this article is to give a brief survey of some singular perturbation problems arising from the van der Waals-Cahn-Hilliard gradient theory of phase transitions (cf. [8, 34]) to study the equilibrium of a system of two immiscible fluids (or two different phases of the same fluid) enclosed in a fixed container.

Assuming that the container is represented by an open, bounded and Lipschitz domain $\Omega \subset \mathbb{R}^{N}$, and that the free energy per unit volume of this system, neglecting the contact energy between the fluid and the container walls, is a prescribed non-convex, non-negative and continuous function $W=W(u)$, of the density distribution $u: \Omega \rightarrow \mathbb{R}$, where $W$ attains the minimum value of zero at exactly two points $\alpha$ and $\beta$ with $\alpha \neq \beta$ (double well potential), the Gibbs criterion (cf. Gurtin [22]) states that stable configurations of this system may be identified as minimizers of its total energy

$$
\int_{\Omega} W(u(x)) d x
$$

among all density distributions $u$ of prescribed total mass $m$

$$
m=\frac{1}{|\Omega|} \int_{\Omega} u(x) d x
$$

where

$$
m=\theta \alpha+(1-\theta) \beta, 0<\theta<1 .
$$

With this formulation, this problem admits infinitely many solutions, namely all piecewise constant measurable functions of the form $u(x)=\alpha \chi_{A}(x)+$ $\beta\left(1-\chi_{A}(x)\right)$ where $A$ is any measurable subset of $\Omega$ with $\mathcal{L}^{N}(A)=\theta \mathcal{L}^{N}(\Omega)$ due to the constraint (1.2). In particular, no restriction is imposed on the shape of the boundary between the sets $A$ and $\Omega \backslash A$. This lack of uniqueness arises because interfaces, i.e, sets of discontinuity points of $u$, are allowed to form without an increase of energy.

A way to curtail this problem consists on the addition of an interfacial energy term directly to the expression of the total energy, and subsequent search for solutions that arise as limiting cases within that theory (see, for instance, Gurtin [21, 23]). This penalization of the formation of interfaces leads to the selection of (physically preferred) solutions in which the fluids arrange themselves so as to minimize the area of the interface separating the two phases.

Alternatively, the van der Waals-Cahn-Hilliard theory is based on the study of perturbed energy functionals of (1.1) by a gradient term, leading to an 
energy of the form

$$
E_{\varepsilon}(u)=\int_{\Omega} f_{\varepsilon}(x, u(x), \nabla u(x)) d x
$$

where

$$
f_{\varepsilon}(x, u, \nabla u)=W(u)+\varepsilon^{2}|\nabla u|^{2},
$$

and the interfacial energy is modeled by the dependence on the density gradient that penalizes the formation of interfaces (cf. Gurtin [22]).

The mathematical problem is then to study the asymptotic behaviour, as $\varepsilon \rightarrow 0^{+}$, of the solutions $u_{\varepsilon}$ of the minimization problems

$$
\min \left\{E_{\varepsilon}(u): \frac{1}{|\Omega|} \int_{\Omega} u(x) d x=m\right\}
$$

and to show, as conjectured by Gurtin (cf. [22]), that (for a subsequence) $u_{\varepsilon}$ converges to a function which takes only the values $\alpha$ and $\beta$ and for which the interface between the sets $\{u=\alpha\}$ and $\{u=\beta\}$ has minimal area.

Our objective is to give a brief account of this asymptotic problem and others of the same type that have been addressed by means of the theory of $\Gamma$-convergence.

The plan of this paper is as follows. In Section 2 we fix notation and give a short overview of functions of bounded variation and $\Gamma$-convergence of functionals. In Section 3 we will present some examples of rescaled functionals of the type (1.4) which, through a $\Gamma$-limit process as the small parameter $\varepsilon \rightarrow 0^{+}$, select a physically reasonable solution for the initial problem. In Section 4 a similar discussion is presented for functionals involving second order terms.

\section{Notation and preliminaries}

Throughout this text, unless otherwise specified, $\Omega \subset \mathbb{R}^{N}, N \geq 2$, will denote an open bounded set with Lipschitz boundary and we will use the following notations:

- $|\Omega|$ denotes the Lebesgue measure of $\Omega$.

- $\mathbb{R}_{+}^{d}:=[0,+\infty)^{d}$.

- $\mathcal{L}^{N}$ and $\mathcal{H}^{N-1}$ stand, respectively, for the $N$-dimensional Lebesgue measure and the $(N-1)$-dimensional Hausdorff measure in $\mathbb{R}^{N}$.

- $\mathcal{M}(\Omega)$ stands for the set of finite Radon measures on $\Omega$.

- $Q$ is the open unit cube in $\mathbb{R}^{N}$ centered at the origin with faces normal to the coordinates axes.

- $B(x, r)$ denotes the open ball centered at $x \in \mathbb{R}^{N}$ with radius $r>0$. 
- $\mathbb{S}^{N-1}:=\left\{x \in \mathbb{R}^{N}:|x|=1\right\}$.

- Given $\nu \in \mathbb{S}^{N-1}$ the set $S_{\nu}$ represents the strip

$$
S_{\nu}:=\left\{x \in \mathbb{R}^{N}:|x \cdot \nu|<\frac{1}{2}\right\}
$$

and $Q_{\nu}$ denotes an open unit cube centered at the origin with two of its faces normal to $\nu$, i.e., if $\left\{\nu_{1}, \ldots, \nu_{N-1}, \nu\right\}$ is an orthonormal basis of $\mathbb{R}^{N}$ then

$Q_{\nu}:=\left\{x \in \mathbb{R}^{N}:|x \cdot \nu|<\frac{1}{2},\left|x \cdot \nu_{i}\right|<\frac{1}{2}, i=1, \ldots, N-1\right\}$.

- $Q_{\nu}\left(x_{0}, r\right):=x_{0}+r Q_{\nu}$ for $x_{0} \in \mathbb{R}^{N}, r>0$ and $\nu \in \mathbb{S}^{N-1}$. If $\left\{e_{1}, \ldots, e_{N}\right\}$ is the canonical basis of $\mathbb{R}^{N}$ then $Q_{e_{N}}\left(x_{0}, r\right)=x_{0}+$ $r Q=: Q\left(x_{0}, r\right)$.

- $C_{\text {per }}(Q)$ represents the set of all continuous and $Q$-periodic functions.

- $\mathrm{Sym}^{d}$ stands for the space of symmetric $d \times d$ matrices.

- $\mathcal{T}^{d \times d \times N}$ is the space of tensors $\Lambda=\left(\Lambda^{1}, \ldots, \Lambda^{N}\right), \Lambda^{i} \in \mathrm{Sym}^{d}$, $i=1, \ldots, N$.

- $\otimes$ and $\odot$ represent, respectively, the usual tensor product and symmetric tensor product of two tensors.

- $C$ denotes a generic positive constant whose value might change from line to line.

We now introduce some definitions and standard facts from the theory of $B V$-functions and we refer to Ambrosio, Fusco \& Pallara [2] for an exhaustive exposition of the subject.

A function $u \in L^{1}\left(\Omega ; \mathbb{R}^{d}\right)$ is said to be of bounded variation, and we write $u \in B V\left(\Omega ; \mathbb{R}^{d}\right.$ ) (or $B V(\Omega)$ for $d=1$ ), if all its first order distributional derivatives $D_{j} u_{i}$ belong to $\mathcal{M}(\Omega)$ for $i=1, \ldots, N$ and $j=1, \ldots, d$. The matrix-valued measure whose entries are $D_{j} u_{i}$ is denoted by $D u$.

Clearly, we have that any $u \in W^{1,1}\left(\Omega ; \mathbb{R}^{d}\right)$ is a $B V$-function with $D u \in$ $L^{1}\left(\Omega ; \mathbb{R}^{d}\right)$ and the measures $D u_{j}^{i}$ are absolutely continuous with respect to the Lebesgue measure.

The space $B V\left(\Omega ; \mathbb{R}^{d}\right)$ is a Banach space when endowed with the norm

$$
\|u\|_{B V}=\|u\|_{L^{1}}+|D u|(\Omega),
$$

where $|D u|$ stands for the total variation measure of $D u$. 
Given $u \in B V\left(\Omega ; \mathbb{R}^{d}\right)$, let $\Omega_{u}$ be the set of points $x \in \Omega$ where the approximate limit of $u$ exists, i.e. such that there exists $z \in \mathbb{R}^{d}$ with

$$
\lim _{\varepsilon \rightarrow 0^{+}} f_{B(x, \varepsilon)}|u(y)-z| d y=0 .
$$

If $x \in \Omega_{u}$ and $z=u(x)$ we say that $u$ is approximately continuous at $x$ (or that $x$ is a Lebesgue point of $u$ ). The function $u$ is approximately continuous $\mathcal{L}^{N}$-a.e. $x \in \Omega_{u}$ and

$$
\mathcal{L}^{N}\left(S_{u}\right)=0
$$

where we denote by $S_{u}$ the set of points where $u$ is not approximately continuous, ie., $S_{u}=\Omega \backslash \Omega_{u}$. We say that $x \in S_{u}$ is an approximate jump point of $u$ if there exists $\nu_{u}(x) \in \mathbb{S}^{N-1}$ and $u^{ \pm}(x) \in \mathbb{R}^{d}$ such that

$$
\lim _{r \rightarrow 0^{+}} \frac{1}{r^{d}}\left(\int_{B^{+}(x, r)}\left|u(y)-u^{+}(x)\right| d y+\int_{B^{-}(x, r)}\left|u(y)-u^{-}(x)\right| d y\right)=0,
$$

with $B^{ \pm}(x, r):=\left\{y \in B(x, r): \pm(y-x) \cdot \nu_{u}(x)>0\right\}$. The triple $\left(\nu_{u}(x), u^{+}(x), u^{-}(x)\right)$ is unique up to a change of sign of $\nu_{u}(x)$ and a permutation of $u^{+}(x)$ and $u^{-}(x)$. The set of approximate jump points is denoted by $J_{u}$.

By the Lebesgue-Radon-Nikodým Theorem, if $u \in B V\left(\Omega ; \mathbb{R}^{d}\right)$ then $D u$ can be split into the sum of two mutually singular measures $D^{a} u$ and $D^{s} u$ (the absolutely continuous part and singular part, respectively, of $D u$ with respect to the Lebesgue measure $\mathcal{L}^{N}$ ). We denote by $\nabla u$ the Radon-Nikodým derivative of $D^{a} u$ with respect to $\mathcal{L}^{N}$, so that we can write

$$
D u=\nabla u \mathcal{L}^{N}\left\lfloor\Omega+D^{s} u .\right.
$$

We recall that an $\mathcal{H}^{N-1}$-measurable set $E \subset \mathbb{R}^{N}$ is said to be a countably $\mathcal{H}^{N-1}$-rectifiable set if it can be covered $\mathcal{H}^{N-1}$-almost everywhere by a countable family of $(N-1)$-dimensional surfaces of class $C^{1}$.

It is well known that if $\Omega \subset \mathbb{R}^{N}$ is open and $u \in B V\left(\Omega ; \mathbb{R}^{d}\right)$, then $J_{u}$ is a countably $\mathcal{H}^{N-1}$-rectifiable set oriented by $\nu_{u}, \mathcal{H}^{N-1}\left(S_{u} \backslash J_{u}\right)=|D u|\left(S_{u} \backslash\right.$ $\left.J_{u}\right)=0$ and $D^{s} u$ can be decomposed as $D^{c} u+D^{j} u$, where $\left|D^{c} u\right|(E)=0$ for every Borel set $E$ with $\mathcal{H}^{N-1}(E)<+\infty$, and

$$
D^{j} u=\left(u^{+}-u^{-}\right) \otimes \nu_{u} \mathcal{H}^{N-1}\left\llcorner J_{u} .\right.
$$

$D^{c} u$ and $D^{j} u$ are called the Cantor part and the jump part of the measure $D u$, respectively.

We also recall that a $\mathcal{L}^{N}$-measurable subset $E \subset \mathbb{R}^{N}$ is a set of finite perimeter in $\Omega$ if the characteristic function $\chi_{E}$ of $E$ is a function of bounded 
variation. In this case, the perimeter of $E$ in $\Omega$ is given by the total variation of $\chi_{E}$ in $\Omega$, i.e., $\operatorname{Per}_{\Omega}(E):=\left|D \chi_{E}\right|(\Omega)$.

Let $E$ be a $\mathcal{L}^{N}$-measurable subset of $\mathbb{R}^{N}$ and $\Omega$ be the largest open set such that $E$ is locally of finite perimeter in $\Omega$, i.e., such that $\chi_{E} \in B V_{\mathrm{loc}}(\Omega)$. The reduced boundary of $E, \partial^{*} E$, is the collection of all points $x_{0} \in \Omega$ such that

(i) $\left|D \chi_{E}\right|\left(B\left(x_{0}, r\right)\right)>0$ for all $r>0$, that is, $x_{0} \in \operatorname{supp}\left|D \chi_{E}\right|$;

(ii) the limit $\nu_{E}\left(x_{0}\right):=\lim _{r \rightarrow 0^{+}} \frac{D \chi_{E}\left(B\left(x_{0}, r\right)\right)}{\left|D \chi_{E}\right|\left(B\left(x_{0}, r\right)\right)}$ exists in $\mathbb{R}^{N}$;

(iii) $\left|\nu_{E}\left(x_{0}\right)\right|=1$.

The function $\nu_{E}: \partial^{*} E \rightarrow \mathbb{S}^{N-1}$ is called the generalized unit inner normal to $E$.

It can be easily checked that $\partial^{*} E$ is a Borel set and that $\nu_{E}$ is a Borel map. By the Besicovitch Derivation Theorem the measure $\left|D \chi_{E}\right|$ is concentrated on $\partial^{*} E$ and $D \chi_{E}=\nu_{E}\left|D \chi_{E}\right|$. In addition, by De Giorgi's Rectifiability Theorem, see [2, Theorem 3.59], $\left|D \chi_{E}\right|$ coincides with $\mathcal{H}^{N-1}\left\llcorner\partial^{*} E\right.$, and for every $x \in \partial^{*} E$ the following properties hold

$$
\begin{aligned}
& \lim _{r \rightarrow 0^{+}} \frac{1}{r^{N-1}} \mathcal{H}^{N-1}\left(\partial^{*} E \cap Q_{\nu_{E}(x)}(x, r)\right)=1 \\
& \lim _{r \rightarrow 0^{+}} \frac{1}{r^{N}} \mathcal{L}^{N}\left(\left\{y \in B(x, r) \backslash E:(y-x) \cdot \nu_{E}(x) \geq 0\right\}\right)=0 \\
& \lim _{r \rightarrow 0^{+}} \frac{1}{r^{N}} \mathcal{L}^{N}\left(\left\{y \in B(x, r) \cap E:(y-x) \cdot \nu_{E}(x) \leq 0\right\}\right)=0
\end{aligned}
$$

(see also Evans \& Gariepy [13, § 5.7.2, Corollary 1]).

Given $\alpha, \beta \in \mathbb{R}^{d}, \alpha \neq \beta$, we denote by $B V(\Omega ;\{\alpha, \beta\})$ the set of all vectorvalued functions $u$ of bounded variation in $\Omega$ such that $u(x) \in\{\alpha, \beta\}$ for $\mathcal{L}^{N}$-a.e. $x \in \Omega$. If $u \in B V(\Omega ;\{\alpha, \beta\})$, that is, $u=\beta \chi_{E}+\alpha \chi_{\Omega \backslash E}$ for some $\mathcal{L}^{N}$-measurable set $E$ of finite perimeter, then $S_{u}$, the reduced boundary $\partial^{*} E$ and the jump set $J_{u}$ of $u$ have the same $\mathcal{H}^{N-1}$-measure in $\Omega$. By $(2.3)$ and (2.4), we also have $\nu_{u}(x)=\nu_{E}(x), u^{+}(x)=\beta$ and $u^{-}(x)=\alpha$, for $\mathcal{H}^{N-1}$-a.e. $x \in \partial^{*} E$.

We end this section by briefly recalling De Giorgi's notion of $\Gamma$-convergence.

Let $X$ denote a metric space. A functional $F: X \rightarrow \overline{\mathbb{R}}$ is said to be the $\Gamma$-limit of the family of functionals $F_{\varepsilon}$ with respect to the metric of $X$ if, for every $u \in X$, the following conditions hold: 
i) for every sequence $\varepsilon_{n} \rightarrow 0^{+}$and for every sequence $u_{n} \rightarrow u$ in $X$ we have

$$
F(u) \leq \liminf _{n \rightarrow+\infty} F_{\varepsilon_{n}}\left(u_{n}\right)
$$

ii) for every sequence $\varepsilon_{n} \rightarrow 0^{+}$there exists a sequence $u_{n} \rightarrow u$ in $X$ such that

$$
\limsup _{n \rightarrow+\infty} F_{\varepsilon_{n}}\left(u_{n}\right) \leq F(u) .
$$

This sequence in ii) is called the recovery sequence for the $\Gamma$-limit.

The notion of $\Gamma$-convergence is especially important when dealing with minimization problems since if $u_{n}$ is a sequence of minimum points of the functionals $F_{\varepsilon_{n}}$, and $u_{n}$ converges to $u$ with respect to the metric of $X$, then $u$ is a minimum point of $F$ and $\lim _{n \rightarrow+\infty} F_{\varepsilon_{n}}\left(u_{n}\right)=F(u)$.

\section{First order problems}

Upon rescaling, the problem (1.6) introduced in Section 1, becomes to study the asymptotic behaviour, as $\varepsilon \rightarrow 0^{+}$, of the solutions $u_{\varepsilon}$ of the minimization problems

where

$$
\min \left\{E_{\varepsilon}(u): \frac{1}{|\Omega|} \int_{\Omega} u(x) d x=m\right\}
$$

$$
E_{\varepsilon}(u)=\frac{1}{\varepsilon} \int_{\Omega} f_{\varepsilon}(x, u(x), \nabla u(x)) d x
$$

with

$$
f_{\varepsilon}(x, u, \nabla u)=W(u)+\varepsilon^{2}|\nabla u|^{2} .
$$

For $N=1$ (one-dimensional case) Gurtin's conjecture was proven by Carr, Gurtin \& Slemrod [8]. Precisely, if $\Omega=(0,1)$, the authors showed that, for small $\varepsilon,(3.1)$ admits only two minimizers and that the minimum value is given by $\varepsilon K_{0}+O\left(e^{-C / \varepsilon}\right)$, where $K_{0}=2 \int_{\alpha}^{\beta} \sqrt{W(s)} d s$ is the surface energy per unit area and $C>0$ is a constant (see also Owen [29]).

For $N>1$ Gurtin's conjecture was proved independently by Modica [27] and Sternberg [32] using $\Gamma$-convergence techniques and following ideas of a previous work by Modica \& Mortola [28]. In particular, considering the family of energies $J_{\varepsilon}: L^{1}(\Omega) \rightarrow \mathbb{R} \cup\{+\infty\}$

$$
J_{\varepsilon}(u)= \begin{cases}\int_{\Omega} \frac{1}{\varepsilon} W(u(x))+\varepsilon|\nabla u(x)|^{2} d x, & \text { if } u \in W^{1,2}(\Omega) \cap \mathcal{V} \\ +\infty, & \text { otherwise }\end{cases}
$$


where

$$
\mathcal{V}=\left\{u \in L^{1}(\Omega): \frac{1}{|\Omega|} \int_{\Omega} u(x) d x=m\right\}
$$

it was shown in [27] (see also [32]) that the $\Gamma$-limit, with respect to the $L^{1}(\Omega)$-topology, of the family $J_{\varepsilon}$ is given by the functional $J_{0}: L^{1}(\Omega) \rightarrow$ $\mathbb{R} \cup\{+\infty\}$

$$
J_{0}(u)= \begin{cases}2 \operatorname{Per}_{\Omega}(\{u=\alpha\}) \int_{\alpha}^{\beta} \sqrt{W(s)} d s, & \text { if } u \in B V(\Omega ;\{\alpha, \beta\}) \cap \mathcal{V} \\ +\infty, & \text { otherwise. }\end{cases}
$$

The properties of $\Gamma$-convergence (see the end of Section 2) guarantee, in particular, that for the given volume fraction $\theta$ in (1.3), preferred solutions of (3.1) are those which exhibit minimal interfacial area.

Generalizations of (3.1) to the coupled case for integrands of the form

$$
f_{\varepsilon}(x, u, \nabla u)=f(x, u, \varepsilon \nabla u),
$$

with $f$ a convex function in the last variable, were obtained by Owen \& Sternberg [30] and Bouchitté [7] who treated the case where $f(x, u, 0)=0$ if and only if $u \in\{\alpha(x), \beta(x)\}$ for $\alpha$ and $\beta$ two Lipschitz continuous functions such that $\alpha(x) \leq \beta(x)$, for all $x \in \Omega$. From the physical point of view, this situation occurs when the temperature inside $\Omega$ is a given function of $x$. Minimizers of (3.1) converge, up to a subsequence, to a function of the form $u=\alpha \chi_{A}+\beta \chi_{\Omega \backslash A}$, where $A \subseteq\{x \in \Omega: \beta(x)>\alpha(x)\}$ is a solution of a certain geometric variational problem.

We also refer to Kohn \& Sternberg [25] where the study of local minimizers for problem (3.1) is addressed.

In the vector-valued setting, where $u: \Omega \rightarrow \mathbb{R}^{d}, N, d>1$, the study of the minimization problem (3.1) with the energies given in (3.3) was undertaken by Fonseca \& Tartar [20]. More precisely, assuming that $W \in W_{\text {loc }}^{1, \infty}\left(\mathbb{R}^{d}\right)$ is a non-negative function such that

$$
W(u)=0 \Leftrightarrow u \in\{\alpha, \beta\},
$$

satisfying the growth condition

$$
\exists C, R>0:|u|>R \Rightarrow W(u) \geq C|u|,
$$

and for which there exist $\gamma, \delta>0$ such that

$$
\begin{aligned}
& |u-\alpha|<\delta \Rightarrow \gamma|u-\alpha|^{2} \leq W(u) \leq \frac{1}{\gamma}|u-\alpha|^{2} \quad \text { and } \\
& |u-\beta|<\delta \Rightarrow \gamma|u-\beta|^{2} \leq W(u) \leq \frac{1}{\gamma}|u-\beta|^{2},
\end{aligned}
$$


the authors proved that the $\Gamma$-limit of the family of functionals $J_{\varepsilon}$ is given by

$$
J_{0}(u)= \begin{cases}\bar{K} \operatorname{Per}_{\Omega}(\{u=\alpha\}), & \text { if } u \in B V(\Omega ;\{\alpha, \beta\}) \cap \mathcal{V} \\ +\infty, & \text { otherwise }\end{cases}
$$

where

$$
\begin{aligned}
& \bar{K}= \\
& 2 \inf \left\{\int_{-1}^{1} \sqrt{W(g(s))}\left|g^{\prime}(s)\right| d s: g \text { is piecewise } C^{1}, g(-1)=\alpha, g(1)=\beta\right\}
\end{aligned}
$$

represents the energy left on the interface as the boundary layer goes to zero. Their proof relies on the study of the properties of a "geodesical distance" defined in terms of the least energy along paths connecting the two wells. The form of $J_{0}$ and the role played by the geodesic curves were independently conjectured by Kohn \& Stenberg [25], who refer also to Mahoney \& Norbury [26]. Given that $\bar{K}$ is a constant, if $u_{\varepsilon}$ is a family of minimum points for problem (3.1) and $u_{\varepsilon} \rightarrow u_{0}$, then, once again, $u_{0} \in B V(\Omega ;\{\alpha, \beta\})$ has minimal interfacial area.

The work in [20] was extended by Baldo [4] to the case where $W$ supports multiple wells (see also Sternberg [33]) and later generalized by Ambrosio [1].

For integrands of the form

$$
f_{\varepsilon}(x, u, \nabla u)=W(u)+\varepsilon^{2} h^{2}(x, \nabla u),
$$

the $\Gamma$-limit, with respect to the $L^{1}\left(\Omega ; \mathbb{R}^{d}\right)$-topology, of the rescaled energies $J_{\varepsilon}: L^{1}\left(\Omega ; \mathbb{R}^{d}\right) \rightarrow \mathbb{R} \cup\{+\infty\}$, where

$$
J_{\varepsilon}(u)= \begin{cases}\int_{\Omega} \frac{1}{\varepsilon} W(u(x))+\varepsilon h^{2}(x, \nabla u(x)) d x, & \text { if } u \in W^{1,2}\left(\Omega ; \mathbb{R}^{d}\right) \\ +\infty, & \text { otherwise }\end{cases}
$$

was identified by Barroso \& Fonseca [6]. In this case the original energy is perturbed via an anisotropic term, whose dependence on the spatial variable $x$ accounts for the fact that the material may be inhomogeneous. Assuming that the density $W$ satisfies a growth condition of the form

$$
C_{1}|u|^{q}-C \leq W(u) \leq C\left(1+|u|^{q}\right), \text { for some } q \geq 2,
$$

that the anisotropic density satisfies the linear growth condition

$$
C_{1}|\xi|-C \leq h(x, \xi) \leq C(1+|\xi|)
$$


and under some other continuity hypotheses, the $\Gamma$-limit of $J_{\varepsilon}$ turns out to be the functional

$$
J_{0}: L^{1}\left(\Omega ; \mathbb{R}^{d}\right) \rightarrow \mathbb{R} \cup\{+\infty\}
$$

given by

$J_{0}(u)= \begin{cases}\int_{\Omega \cap \partial^{*}\{u=\alpha\}} K(x, \alpha, \beta, \nu(x)) d H^{N-1}(x), & \text { if } u \in B V(\Omega ;\{\alpha, \beta\}) \\ +\infty, & \text { otherwise, }\end{cases}$

where $\nu(x)$ is the outward unit normal to the interface $\Omega \cap \partial^{*}\{u=\alpha\}$ and the surface energy density $K$ is defined by

$$
\begin{aligned}
& K(x, \alpha, \beta, \nu)= \\
& \quad \inf \left\{\int_{Q_{\nu}} L W(w(y))+\frac{1}{L}\left[h^{\infty}(x, \nabla w(y))\right]^{2} d y: L>0, w \in \mathcal{A}(\nu)\right\}
\end{aligned}
$$

where, for every $\nu \in \mathbb{S}^{N-1}$, the class of admissible density functions is given by

$$
\begin{aligned}
& \mathcal{A}(\nu):= \\
& \left\{w \in W_{\mathrm{loc}}^{1,2}\left(S_{\nu} ; \mathbb{R}^{d}\right): w(y)=\alpha \text { if } y \cdot \nu=-\frac{1}{2}, \quad w(y)=\beta \text { if } y \cdot \nu=\frac{1}{2},\right. \\
& \left.w(y)=w\left(y+l \nu_{i}\right), \text { for all } y \in S_{\nu}, \quad i=1, \ldots, N-1, \text { and } l \in \mathbb{Z}\right\} .
\end{aligned}
$$

As usual, the function $h^{\infty}$ in (3.4) denotes the recession function of $h$ given by

$$
h^{\infty}(x, \xi)=\limsup _{t \rightarrow+\infty} \frac{h(x, t \xi)}{t}
$$

which gives us the asymptotic behaviour of $h$ at $+\infty$.

Assuming further that $W \in W_{\text {loc }}^{1, \infty}\left(\mathbb{R}^{d}\right)$ is such that there exist $\gamma, \delta>0$ with

$$
\begin{aligned}
& |u-\alpha|<\delta \Rightarrow \gamma|u-\alpha|^{q} \leq W(u) \leq \frac{1}{\gamma}|u-\alpha|^{q} \quad \text { and } \\
& |u-\beta|<\delta \Rightarrow \gamma|u-\beta|^{q} \leq W(u) \leq \frac{1}{\gamma}|u-\beta|^{q},
\end{aligned}
$$


and that $h^{2}(x, \cdot)$ is quasiconvex, the minimization problem

$$
\begin{aligned}
\min \left\{\int_{\Omega} W(u(x))+\right. & \varepsilon^{2} h^{2}(x, \nabla u(x)) d x: \\
u & \left.\in W^{1,2}\left(\Omega ; \mathbb{R}^{d}\right), \frac{1}{|\Omega|} \int_{\Omega} u(x) d x=m\right\}
\end{aligned}
$$

is also treated in [6]. The authors begin by showing that any sequence $v_{n}$ of bounded energy, $J_{\varepsilon_{n}}\left(v_{n}\right) \leq C<+\infty$, is relatively compact in $L^{1}\left(\Omega ; \mathbb{R}^{d}\right)$. Thus, any sequence of solutions of (3.6), whose existence is guaranteed by the hypotheses above, has a subsequence converging to the solution of the initial problem which minimizes the integral over the interface of the surface energy density $K(x, \alpha, \beta, \nu)$. The proof of Barroso \& Fonseca's result is based on the blow-up method introduced by Fonseca \& Müller and on a slicing technique which allows to replace a sequence near the boundary of a cube by a new sequence satisfying an appropriate boundary condition but in such a way that the total energy does not increase.

Under similar hypotheses Fonseca \& Popovici [19] studied the vector-valued and coupled case for integrands of the form

$$
f_{\varepsilon}(x, u, \nabla u)=f(x, u, \varepsilon \nabla u)
$$

where $f(x, u, 0)=0 \Leftrightarrow u \in\{\alpha, \beta\}$ and

$$
\frac{1}{C}\left(|u|^{q}+|\xi|^{2}-C\right) \leq f(x, u, \xi) \leq C\left(1+|u|^{q}+|\xi|^{2}\right)
$$

for some $q \geq 2, C>0$. The expression obtained for the $\Gamma$-limit, in this case, is exactly as the one in [6] with the surface energy density replaced by

$$
K(x, \alpha, \beta, \nu)=\inf \left\{\int_{Q_{\nu}} \frac{1}{s} f(x, \xi(y), s \nabla \xi(y)) d y: s>0, \xi \in \mathcal{A}(\nu)\right\} .
$$

\section{Second order problems}

In the context of elastic solid-to-solid phase transitions (see Ball \& James [5], Coleman, Marcus \& Mizel [11] and Kohn \& Müller [24]), Conti, Fonseca \& Leoni [12] addressed the characterization of the $\Gamma$-limit for functionals involving second order integrands of the form

$$
f_{\varepsilon}(x, u, \nabla u)=W(\nabla u)+\varepsilon^{2}\left|\nabla^{2} u\right|^{2}
$$


that, after rescaling, led to the study of the family $J_{\varepsilon}: L^{1}\left(\Omega ; \mathbb{R}^{d}\right) \rightarrow \mathbb{R} \cup\{+\infty\}$

$$
J_{\varepsilon}(u)= \begin{cases}\int_{\Omega} \frac{1}{\varepsilon} W(\nabla u(x))+\varepsilon\left|\nabla^{2} u(x)\right|^{2} d x, & \text { if } u \in W^{2,2}\left(\Omega ; \mathbb{R}^{d}\right) \\ +\infty, & \text { otherwise. }\end{cases}
$$

To simplify the problem the authors neglected the frame-indifference constraint on $W$ and simply assume that $W$ is a continuous function such that

$$
\{W=0\}=\{A, B\}, \quad A-B=a \otimes \nu,
$$

for some $a \in \mathbb{R}^{d} \backslash\{0\}$ and $\nu \in \mathbb{S}^{N-1}$, since, in view of Hadamard's compatibility condition, the two matrices $A$ and $B$ must be rank-one connected. Under the hypothesis that there exists $C>0$ such that

$$
W(\xi) \geq C|\xi|-\frac{1}{C} \text { for all } \xi \in \mathbb{R}^{d \times N},
$$

a compactness result is proved showing that (up to a subsequence) the difference between any sequence with bounded energy and its average over $\Omega$, converges to a function $u \in W^{1,1}\left(\Omega ; \mathbb{R}^{d}\right)$ with $\nabla u \in B V(\Omega ;\{A, B\})$. Moreover, assuming that for all $\xi=\left(\xi_{1}, \ldots, \xi_{N}\right) \in \mathbb{R}^{d \times N}, \xi_{i} \in \mathbb{R}^{d}$,

$$
\begin{gathered}
W(\xi) \rightarrow \infty \text { as }|\xi| \rightarrow \infty, \\
W(\xi) \geq W\left(0, \xi_{N}\right)
\end{gathered}
$$

and that $W$ is differentiable at $A$ and $B$, given $u \in W^{1,1}\left(\Omega ; \mathbb{R}^{d}\right)$ with $\nabla u \in$ $B V(\Omega ;\{A, B\})$ it was proved in [12] that

$$
\Gamma\left(L^{1}\right)-\lim _{\varepsilon \rightarrow 0^{+}} J_{\varepsilon}(u)=K \operatorname{Per}_{\Omega}(\mathrm{E})
$$

where $\nabla u=\left(1-\chi_{E}(x)\right) A+\chi_{E}(x) B$ for $\mathcal{L}^{N}$-a.e $x \in \Omega$ with

$$
\begin{aligned}
K=\inf \left\{\int_{\mathrm{L}}^{-\mathrm{L}} \mathrm{W}(0, \mathrm{~g}(\mathrm{~s}))+\left|\mathrm{g}^{\prime}(\mathrm{s})\right|^{2} \mathrm{ds}: \mathrm{L}\right. & >0, \\
g & \text { piecewise } \left.\mathrm{C}^{1}, \mathrm{~g}(-\mathrm{L})=-\mathrm{a}, \mathrm{g}(\mathrm{L})=\mathrm{a}\right\},
\end{aligned}
$$

which is similar to a corresponding expression obtained in [20] due to the one-dimensional character of (4.2).

The main difficulty of their proof relies on the construction of a recovery sequence (see Theorem 1.3 in [12]; and also Theorem 1.4 where a similar result was obtained under weaker assumptions than (4.2)). Precisely, if the admissible fields are such that $\nabla u$ has a layered structure with two interfaces, then it is possible to construct an effective sequence near each 
interface but the task of "gluing" together the two sequences on a suitable low-energy intermediate layer is delicate.

In the two-dimensional case and when $W$ vanishes on the unit circle (4.1) reduces to the so-called Eikonal functional arising in the theory of liquid crystals as well as in blistering of delaminated thin films (see Fonseca \& Leoni [15] for references on these problems and on other generalizations).

We also mention the work of Chermisi, Dal Maso, Fonseca \& Leoni [9], where, for scalar functions $u$, the authors addressed a model on pattern formation based on the Ginzburg-Landau energy

$$
\int_{\Omega} W(u(x))-q|\nabla u(x)|^{2}+\left|\nabla^{2} u(x)\right|^{2} d x
$$

(see [11] and Seul \& Andelman [31]) by considering a perturbed second order energy of the form

$$
f_{\varepsilon}\left(x, u, \nabla u, \nabla^{2} u\right)=W(u)-q \varepsilon^{2}|\nabla u|^{2}+\varepsilon^{4}\left|\nabla^{2} u\right|^{2}, \quad q>0
$$

(see also Fonseca \& Mantegazza [16] for the case $q=0$, Cicalese, Spadaro \& Zeppieri [10] for $q>0$ in the one-dimensional case, and Hilhorst, Peletier \& Schätzle [14] for the case $q<0$ where $\left|\nabla^{2} u\right|^{2}$ is replaced by $|\triangle u|^{2}$ ). Using $\Gamma$ convergence techniques, the authors characterized the singular perturbation limit of the family of rescaled energies

$$
E_{\varepsilon}(u)=\int_{\Omega} \frac{1}{\varepsilon} W(u(x))-q \varepsilon|\nabla u(x)|^{2}+\varepsilon^{3}\left|\nabla^{2} u(x)\right|^{2} d x
$$

where $\Omega$ is a bounded, open subset of $\mathbb{R}^{N}$ with $C^{1}$ boundary, $u: \Omega \subseteq \mathbb{R}^{N} \rightarrow \mathbb{R}$ and $W: \mathbb{R} \rightarrow[0,+\infty)$ is a continuous function satisfying

$$
\begin{gathered}
W(s)=0 \Leftrightarrow s \in\{-1,1\}, \\
W(s) \geq(|s|-1)^{2}, \quad \forall s \in \mathbb{R}
\end{gathered}
$$

and

$W(s) \leq C_{0} W(t)+C_{0}$, for all $s, t \in \mathbb{R}$ such that $|s| \leq|t|$ and for some $C_{0} \geq 1$.

A major difficulty in this analysis is that the energy may have a negative term. To overcome this, an interpolation result is proved, namely that there exists $q^{*}>0$, independent of $\Omega$, such that for all $-\infty<q<\frac{q^{*}}{N}$, there exists $\varepsilon_{0}=\varepsilon_{0}(\Omega, q)$ such that

$$
q \varepsilon^{2} \int_{\Omega}|\nabla u(x)|^{2} d x \leq \int_{\Omega} W(u(x))+\varepsilon^{4}|\nabla u(x)|^{2} d x
$$


for all $0<\varepsilon<\varepsilon_{0}$ and for all $u \in H^{1}(\Omega)$. For $-\infty<q<\frac{q^{*}}{N}$ and under the above hypotheses, the authors showed that the sequence of functionals

$$
J_{\varepsilon}(u)= \begin{cases}E_{\varepsilon}(u), & \text { if } u \in H^{2}(\Omega) \\ +\infty, & \text { if } u \in L^{2}(\Omega) \backslash H^{2}(\Omega)\end{cases}
$$

$\Gamma$-converges, as $\varepsilon \rightarrow 0^{+}$, to the functional $J_{0}: L^{2}(\Omega) \rightarrow \mathbb{R} \cup\{+\infty\}$ defined by

$$
J_{0}(u)= \begin{cases}m_{N} \operatorname{Per}_{\Omega}(\{u=1\}), & \text { if } u \in B V(\Omega ;\{-1,1\}) \\ +\infty, & \text { if } u \in L^{2}(\Omega) \backslash B V(\Omega ;\{-1,1\})\end{cases}
$$

where

$$
\begin{array}{r}
m_{N}=\inf \left\{\int_{Q} \frac{1}{\varepsilon} W(w(x))-q \varepsilon|\nabla w(x)|^{2}+\varepsilon^{3}\left|\nabla^{2} w(x)\right|^{2} d x:\right. \\
0<\varepsilon \leq 1, w \in \mathcal{A}\}>0
\end{array}
$$

and

$$
\begin{aligned}
\mathcal{A}=\left\{w \in H_{\mathrm{loc}}^{2}\left(\mathbb{R}^{N}\right): w(x)=-1 \text { near } x \cdot e_{N}=-\frac{1}{2}, w(x)=1\right. \\
\left.\quad \text { near } x \cdot e_{N}=\frac{1}{2}, w(x)=w\left(x+e_{i}\right), \forall x \in \mathbb{R}^{N}, i=1, \ldots, N-1\right\} .
\end{aligned}
$$

We conclude this survey by mentioning some recent results obtained by Baía, Barroso, Chermisi \& Matias [3] where the authors addressed the asymptotic behaviour, as a small parameter $\varepsilon$ tends to zero, of a sequence of functionals of the form

$$
E_{\varepsilon}(u)=\frac{1}{\varepsilon} \int_{\Omega} f\left(x, u(x), \varepsilon \nabla u(x), \varepsilon^{2} \nabla^{2} u(x)\right) d x
$$

obtained as a singular perturbation of a non-convex second order functional of the type

$$
\int_{\Omega} f\left(x, u(x), \nabla u(x), \nabla^{2} u(x)\right) d x
$$

where $f\left(\cdot, u(\cdot), \nabla u(\cdot), \nabla^{2} u(\cdot)\right)$ represents the free energy of a mixture of $d$ fluids $(d \in \mathbb{N}, d \geq 2)$, occupying a fixed container $\Omega \subset \mathbb{R}^{N}(N \in \mathbb{N}, N \geq 2)$, and is a function of the density $u=\left(u_{1}, \ldots, u_{d}\right)$ and its first and second order derivatives. The bulk energy density $f$ is assumed to be continuous, positive and such that for all $x \in \Omega$ the function $f(x, \cdot, \mathrm{O}, \mathrm{O})$ achieves its minimum value zero at exactly two vectors $\alpha, \beta \in \mathbb{R}_{+}^{d}, \alpha \neq \beta$. In addition, each component $u_{i}$ (the density of the ith-ingredient of the mixture) is considered 
to be nonnegative and the total amount of bulk material is assumed to be preserved, i.e., $u$ belongs to the following set

$$
\begin{aligned}
\mathcal{V}:=\left\{u: \int_{\Omega} u(x) d x=V, V \in \mathbb{R}_{+}^{d},\right. \\
\left.\quad|\Omega| \min \left(\alpha^{i}, \beta^{i}\right)<V^{i}<|\Omega| \max \left(\alpha^{i}, \beta^{i}\right), i=1, \ldots, d\right\},
\end{aligned}
$$

where $V^{i}, \alpha^{i}$ and $\beta^{i}$ are the $i$-th components of $V, \alpha$ and $\beta$, respectively. Assuming there exists a continuous function $g: \bar{\Omega} \times \mathbb{R}_{+}^{d} \rightarrow[0,+\infty)$ such that

$$
\frac{1}{C}\left(g(x, u)+|\xi|^{2}+|\Lambda|^{2}\right) \leq f(x, u, \xi, \Lambda) \leq C\left(g(x, u)+|\xi|^{2}+|\Lambda|^{2}\right)
$$

for all $(x, u, \xi, \Lambda) \in \Omega \times \mathbb{R}_{+}^{d} \times \mathbb{R}^{d \times N} \times \mathcal{T}^{d \times d \times N}$, where $g$ satisfies

$$
\frac{1}{C}|u|^{q}-C \leq g(x, u) \leq C\left(1+|u|^{q}\right)
$$

for some $q \geq 2$, some $C>0$ and for all $(x, u) \in \bar{\Omega} \times \mathbb{R}_{+}^{d}$, and under some technical continuity conditions, they studied, via $\Gamma$-convergence techniques, the family of minimum problems

$$
\min \left\{\frac{1}{\varepsilon} \int_{\Omega} f\left(x, u(x), \varepsilon \nabla u(x), \varepsilon^{2} \nabla^{2} u(x)\right) d x, \quad u \in \mathcal{V}\right\}
$$

and showed that the family of functionals

$$
J_{\varepsilon}(u):= \begin{cases}\frac{1}{\varepsilon} \int_{\Omega} f\left(x, u(x), \varepsilon \nabla u(x), \varepsilon^{2} \nabla^{2} u(x)\right) d x, & u \in W^{2,2}\left(\Omega ; \mathbb{R}_{+}^{d}\right) \cap \mathcal{V}, \\ +\infty, & \text { otherwise }\end{cases}
$$

$\Gamma$-converges, with respect to the $L^{1}\left(\Omega ; \mathbb{R}_{+}^{d}\right)$-convergence, to the functional $J_{0}: L^{1}\left(\Omega ; \mathbb{R}_{+}^{d}\right) \rightarrow \mathbb{R} \cup\{+\infty\}$ defined by

$$
J_{0}(u):= \begin{cases}\int_{J_{u}} \sigma\left(x, \nu_{u}(x)\right) d \mathcal{H}^{N-1}(x), & u \in B V(\Omega ;\{\alpha, \beta\}) \cap \mathcal{V}, \\ +\infty, & \text { otherwise, }\end{cases}
$$

where $\sigma: \Omega \times \mathbb{S}^{N-1} \rightarrow[0,+\infty)$ is given by

$\sigma(x, \nu):=\inf \left\{\int_{Q_{\nu}} \frac{1}{t} f\left(x, w(y), t \nabla w(y), t^{2} \nabla^{2} w(y)\right) d y: t>0, w \in \mathcal{A}(\nu)\right\}$, 
and where for every $\nu \in \mathbb{S}^{N-1}$, the class $\mathcal{A}(\nu)$ of admissible density functions, is defined by

$$
\begin{aligned}
& \mathcal{A}(\nu):=\left\{w \in W_{\mathrm{loc}}^{2,2}\left(S_{\nu} ; \mathbb{R}_{+}^{d}\right): w(y)=\alpha \text { if } y \cdot \nu=-\frac{1}{2}, w(y)=\beta\right. \text { if } \\
& \left.y \cdot \nu=\frac{1}{2}, w(y)=w\left(y+l \nu_{i}\right), \text { for all } y \in S_{\nu}, \quad i=1, \ldots, N-1, \text { and } l \in \mathbb{Z}\right\} .
\end{aligned}
$$

The proof of this result relies on the blow-up method, introduced by Fonseca \& Müller (see e.g [17] and [18]), which allows to consider the case where $\Omega$ is a small cube and the target function has planar interface, together with periodicity arguments and the Riemann-Lebesgue Lemma. A slicing argument is also used in order to modify a sequence near the boundary of the cube without increasing the total energy. The construction of a recovery sequence to prove the upper bound inequality for the $\Gamma$-limit is done in several steps according to the geometry of $\Omega$ and to the interface of the target function. In each of these steps, the recovery sequence must be made to comply with the volume constraint which leads to some difficulties in the proofs.

Due to a compactness result, and by the properties of $\Gamma$-convergence, a sequence of minimizers of the functionals $J_{\varepsilon}$ defined in (4.5), assuming they exist, will converge (up to a subsequence) to a minimizer of the limiting functional $J_{0}$ in (4.6).

Acknowledgements. The research of M. Baía and J. Matias was partially supported by the Fundação para a Ciência e a Tecnologia (Portuguese Foundation for Science and Technology) through CAMGSD and the project UTA-CMU/MAT/0005/2009. The research of A. C. Barroso was partially supported by Fundação para a Ciência e a Tecnologia (Portuguese Foundation for Science and Technology) through the projects PEst OE/MAT/UI0209/2011, PTDC/MAT109973/2009 and UTA-CMU/MAT $/ 0005 / 2009$.

\section{References}

[1] Ambrosio L., Metric space valued functions of bounded variation, Ann. Scuola Norm. Sup. Pisa Cl. Sci. (4), 17 (1990), No. 3, 439-478.

[2] Ambrosio L., Fusco N. and Pallara D., Functions of bounded variation and free discontinuity problems, Oxford Mathematical Monographs. Oxford: Clarendon Press, 2000.

[3] Baía M., Barroso A. C., Chermisi M. and Matias J., Coupled singular perturbations for phase transitions in the presence of surfactants. Nonlinearity 26 (2013) 1271-1311, 2012. 
[4] Baldo S., Minimal interface criterion for phase transitions in mixtures of CahnHilliard fluids, Ann. Inst. Henri Poincaré. Anal. Non Linéaire, 7 (1990), No. 2, 67-90.

[5] Ball, J. M. and James, R. D., Fine phase mixtures as minimizers of energy, Arch. Ration. Mech. Anal., 100 (1987), 13-52.

[6] Barroso A.C. and Fonseca I., Anisotropic singular perturbations - the vectorial case, Proceedings of the Royal Society of Edinburgh, Section A, 124 (1994), 527-571.

[7] Bouchitté G., Singular perturbations of variational problems arising from a twophase transition model, Appl. Math. Optim., 21 (1990), 289-314.

[8] Carr J., Gurtin M. and Slemrod M., Structured phase transitions on a finite interval, Arch. Rational Mech. Anal., 86 (1984), 317-351.

[9] Chermisi M., Dal Maso G., Fonseca I. and Leoni G., Singular perturbation models in phase transition for second order materials, Indiana Univ. Math. J.. To appear.

[10] Cicalese M., Spadaro E. N. and Zeppieri C. I., Asymptotic analysis of a secondorder singular perturbation model for phase transitions. To appear.

[11] Coleman B. D., Marcus M. and Mizel V. J., On the thermodynamics of periodic phases, Arch. Ration. Mech. Anal., 117 (1992), No. 4, 321-347.

[12] Conti S., Fonseca I. and Leoni G., A $\Gamma$-convergence result for the two-gradient theory of phase transitions, Commun. Pure Appl. Math., 55 (2002), No. 7, 857936.

[13] Evans L. C. and Gariepy R. F., Measure theory and fine properties of functions, Studies in Advanced Mathematics. Boca Raton: CRC Press, 1992.

[14] Hilhorst L., Peletier A. and Schatzle R., $\Gamma$-Limit for the extended FisherKolmogorov Equation, Proc. Roy. Soc. Edinburgh Sect. A, 132 (2002), No. 1, 141-162.

[15] Fonseca I. and Leoni G., Higher order variational problems and phase transitions in nonlinear elasticity, CNA preprint, 01-CNA-020, 2001.

[16] Fonseca I. and Mantegazza C., Second order singular perturbation models for phase transitions, SIAM J. Math. Anal., 31 (2000), No. 5, 1121-1143.

[17] Fonseca I. and Müller S., Quasi-convex integrands and lower semicontinuity in $L^{1}$, SIAM J. Math. Analysis, 23 (1992), 1081-1098.

[18] Fonseca I. and Müller S., Relaxation of quasiconvex functionals in $B V\left(\Omega, \mathbb{R}^{N}\right)$ for integrands $f(x, u, \nabla u)$, Arch. Ration. Mech. Anal, 123 (1993), No. 1 1-49.

[19] Fonseca I. and Popovici C., Coupled singular perturbations for phase transitions, Asymptotic Anal., 44 (2005), No. 3-4, 299-325.

[20] Fonseca I. and Tartar L., The gradient theory of phase transitions for systems with two potential wells, Proc. Roy. Soc. Edinburgh Sect. A, 111 (1989), 89-102.

[21] Gurtin M., On a theory of phase transitions with interfacial energy, Arch. Rational Mech. Anal., 87 (1984), 187-212.

[22] Gurtin M., Some results and conjectures in the gradient theory of phase transitions, Institute for Mathematics and Its Applications, University of Minnesota, Preprint no 156, 1985.

[23] Gurtin M., On phase transitions with bulk, interfacial and boundary energy, Arch. Rational Mech. Anal., 96 (1986), 243-264.

[24] Kohn V. R. and Müller S., Surface energy and microstructure in coherent phase transitions, Comm. Pure Appl. Math., 47 (1994), 405-435.

[25] Kohn V. R. and Sternberg P., Local minimizers and singular perturbations, Proc. Royal Soc. Edinburgh Sect. A, 111 (1989), 69-84.

[26] Mahoney J. J. and Norbury J., Asymptotic location of nodal lines using geodesic theory, J. Austral. Math. Soc. Ser. B, 27 (1986), 259-280. 
[27] Modica L., The gradient theory of phase transitions and the minimal interface criterion, Arch. Rational Mech. Anal., 98 (1987), No. 2, 123-142.

[28] Modica L. and Mortola S., Un esempio di $\Gamma$-convergenza, Boll. Un. Mat. Ital. B, 14 (1977), No. 2, 285-299.

[29] Owen N. C., Existence and stability of necking deformations for nonlinearly elastic rods, Arch. Rational Mech. Anal., 98 (1987), 357-383.

[30] Owen N. and Sternberg P., Nonconvex variational problems with anisotropic perturbations, Nonlinear Anal., 16 (1991), 705-719.

[31] Seul M. and Andelman D., Domain shapes and patterns - the phenomenology of modulated phases, Science, 267 (1995), No. 5197, 476-483.

[32] Sternberg P., The effect of a singular perturbation on nonconvex variational problems, Arch. Rational Mech. Anal., 101 (1988), No. 3, 209-260.

[33] Sternberg P., Vector-valued local minimizers of nonconvex variational problems, Rocky Mountain J. Math., 21 (1991), 799-807.

[34] van der Waals D. J., The thermodynamic theory of capillarity under the hypothesis of a continuous variation in density, Journal of Statistical Physics, 20 (1973), 200-244 (translation by J. S Rowlinson). 